BULLETIN OF THE

AMERICAN MATHEMATICAL SOCIETY

Volume 79, Number 4, July 1973

\title{
THE PROBABILITY OF CONNECTEDNESS OF A LARGE UNLABELLED GRAPH ${ }^{1}$
}

\author{
BY E. M. WRIGHT
}

Communicated by Gian-Carlo Rota, December 18, 1972

An $(n, q)$ graph is one with $n$ nodes and $q$ edges, in which any two different nodes are or are not joined by a single edge. We write $T=T(n, q)$ for the number of different $(n, q)$ graphs with unlabelled nodes and $t$ for the number of these graphs which are connected, so that $\beta=t / T$ is the probability that an unlabelled $(n, q)$ graph is connected. We write $F, f$ and $\alpha$ for the corresponding numbers for $(n, q)$ graphs whose nodes are labelled. We write also $N=n(n-1) / 2, B(h, k)=h ! /\{k !(h-k) !\}$ and $\gamma=(2 q-n \log n) / n$. Clearly $q \leqq N$. In what follows, $A$ (not always the same at each occurrence) is a fixed positive number at our choice and all statements are true only for $n>n_{0}, q>q_{0}$, where $n_{0}$ and $q_{0}$ depend on the $A$.

Erdös and Renyi [1] put $q=[n(\log n+a) / 2]$, where $a$ is independent of $n$ and $q$, and showed that, for these $q$, we have

$$
\alpha \rightarrow \exp \left(e^{-a}\right)
$$

as $n \rightarrow \infty$. For given $n$, it can be shown trivially that $\alpha$ increases steadily (in the nonstrict sense) as $q$ increases. Hence, from (1), it can be at once deduced that, as $n \rightarrow \infty$, we have $\alpha \sim \exp \left(e^{-\gamma}\right)$ and, in particular, that

$$
\alpha \rightarrow 1 \quad(\gamma \rightarrow+\infty), \quad \alpha \rightarrow 0 \quad(\gamma \rightarrow-\infty) .
$$

Elsewhere [4] I have shown that, if $\gamma \rightarrow+\infty$, then $f$ has an asymptotic expansion of which the first two terms are

$$
f=B(N, q)-n B(N-n+1, q)-\cdots .
$$

Now $F=B(N, q)$ and

$$
\frac{n B(N-n+1, q)}{B(N, q)}=n \prod_{s=0}^{q-1} \frac{N-n+1-s}{N-s} \leqq n(N-n+1)^{q} N^{-q}
$$

and the logarithm of this is less than $\log n-\{q(n-1) / N\}=-\gamma$. Hence my result leads to $\alpha=1-O\left(e^{-\gamma}\right)$, a statement which is only nontrivial

AMS (MOS) subject classifications (1970). Primary 05C30.

Key words and phrases. Unlabelled graphs, asymptotic enumeration, connectedness, probability of connectedness.

${ }_{1}^{1}$ The research reported herein was sponsored in part by the United States Government. 
when $\gamma \rightarrow+\infty$. Thus, for this range of $q$, I obtain a much more detailed result than Erdös and Renyi. On the other hand, my method (depending on Gilbert's [2] generating functions identity) appears incapable of extension to obtain (1), as indeed Erdös and Renyi remark.

My first theorem here gives a result for $\beta$ corresponding to, but differing from, Erdös and Renyi's result for $\alpha$. The proof depends heavily on the results of [5] and [7].

THEOREM 1. As $n \rightarrow \infty$, we have

$$
\begin{array}{ll}
\beta \sim 1-e^{-\gamma} & (A<\gamma<A), \\
\beta \rightarrow 0 & (\overline{\lim \gamma \leqq 0),} \\
\beta \rightarrow 1 & (\gamma \rightarrow+\infty) .
\end{array}
$$

These results are in striking contrast to Erdös and Renyi's. They imply that, when $-A<\gamma<A$, a substantially higher proportion of the labelled graphs are connected than of the unlabelled, at least in the limit as $n \rightarrow \infty$.

But there is another, and much more interesting difference in the proof required when $\beta \rightarrow 0$ or $\beta \rightarrow 1$. Erdös and Renyi [1] did not need to consider the corresponding cases for $\alpha$ since, for fixed $n$, the number $\alpha$ increases (nonstrictly) with $q$. No such result is known for $\beta$ and indeed, as I showed in [6], no such result is true.

The behavior of $\beta$ for fixed $n$ as $q$ increases presents an interesting problem. Obviously $\beta=0$ for $q \leqq n-2$ and $\beta=1$ for $N-n+2$ $\leqq q \leqq N$. What appears to be true otherwise (by calculations based on the table [3]) is that, for fixed $n \geqq 6$ and some $q_{1}=q_{1}(n)$, we have

$$
\begin{array}{ll}
\beta(n, q)<\beta(n, q+1) & \left(n-2 \leqq q<q_{1}\right), \\
\beta(n, q)>\beta(n, q+1) & \left(q_{1} \leqq q \leqq N-n\right) .
\end{array}
$$

All that I can prove, however, is the following theorem.

THEOREM 2. For $n>n_{0}$ and some $q_{1}=q_{1}(n)$, we have

$$
\begin{array}{ll}
\beta(n, q)<\beta(n, q+1) & \left(n(A+\log n) / 2<q<q_{1}\right), \\
\beta(n, q)>\beta(n, q+1) & \left(q_{1} \leqq q \leqq N-n\right) .
\end{array}
$$

We can calculate the integer $q_{1}$ with a possible error of 1 .

It is surprising that we can define so precisely the range of validity of the unexpected result (3). On the other hand, I cannot prove (2) for $\gamma \leqq 0$, i.e. for $2 q \leqq n \log n$, although the tables [3] and common sense (that dubious guide) combine to indicate that it must be true. In fact, the proof of (2) for $N / 2 \leqq q<q_{1}$ is easier than that for $q \leqq N / 2$ and, in particular, my present proof of (2) for $A<\gamma<A$ is not at all simple. 


\section{REFERENCES}

1. P. Erdös and A. Renyi, On random graphs. I, Publ. Math. 6 (1959), 290-297. MR 22 \# 10924.

2. E. N. Gilbert, Enumeration of labelled graphs, Canad. J. Math 8 (1956), 405-411.

3. M. L. and P. R. Stein, Enumeration of linear graphs and connected linear graphs up to $P=18$ points, Los Alamos Scientific Laboratory, 1963.

4. E. M. Wright, Asymptotic enumeration of connected graphs, Proc. Roy. Soc. Edinburgh A68 (1970), 298-308.

5. - Graphs on unlabelled nodes with a given number of edges, Acta Math. 126 (1970), 1-9. MR 42 \# 2975.

6. - The probability of connectedness of an unlabelled graph can be less for more edges, Proc. Amer. Math. Soc. 35 (1972), 21-25.

7. The number of unlabelled graphs with many nodes and edges, Bull. Amer. Math. Soc. 78 (1972), 1032-1034.

Principal's Office, University of Aberdeen, Aberdeen, United Kingdom 